Itinéraires Itinéraires

Littérature, textes, cultures

2012-3 | 2013

Lire les villes marocaines

\title{
Matière de Fès : littérature, cinérgie et cinécriture
}

\section{Abdelkrim Chiguer}

\section{OpenEdition}

\section{Journals}

Édition électronique

URL : http://journals.openedition.org/itineraires/974

DOI : 10.4000 /itineraires.974

ISSN : 2427-920X

Éditeur

Pléiade

Édition imprimée

Date de publication : 1 juillet 2013

Pagination : 95-110

ISBN : 978-2-343-01183-7

ISSN : $2100-1340$

Référence électronique

Abdelkrim Chiguer, « Matière de Fès : littérature, cinérgie et cinécriture », Itinéraires [En ligne], 2012-3 | 2013, mis en ligne le 01 décembre 2012, consulté le 20 avril 2019. URL : http:// journals.openedition.org/itineraires/974 ; DOI : 10.4000/itineraires.974

Itinéraires est mis à disposition selon les termes de la licence Creative Commons Attribution - Pas d'Utilisation Commerciale - Pas de Modification 4.0 International. 


\title{
Matière de Fès : littérature, cinérgie et cinécriture
}

\begin{abstract}
Walled up because of an overused binarism, intra-muros $v s$ extra-muros, ordre $v s$ chaos, the medina appears in Aya dans les villes, a collection of short stories, material made of stones, letters, but as well still and animated images. The medina allows to raise an hypothesis: poetics are linked less to architecture, painting, ut picture poesis, than to a wise organization of time, and to movie-making. Fes urbanity displays itself like a form of hybridism, which fragments of time, left over for a while, hold out against any definitive reproduction.
\end{abstract}

Keywords : poetics, urbanity, time, cinergy, space, evolution Mots clés : poétique, urbanité, temps, cinérgie, espace, devenir

L'image s'ouvre à la temporalité, par laquelle le cinéma montre dans des mouvements d'appareils son cœur mécanique et son cœur onirique; à travers lesquels prend corps un continent dont la littérature avait éveillé les fantômes. La question n'est pas posée de savoir si le cinéma est un art, une production industrielle ou un ensemble documentaire. Il a été et est tout cela successivement. C'est un fait d'auteur et un fait anonyme.

(Jean-Louis Schefer, Du monde et du mouvement des images, Paris, Cahiers du cinéma, 1997, p. 5).

Les notions de cinérgie et de cinécriture, qui apparaissent dès le titre de cette étude, reviendront souvent dans les pages qui suivent. Nous tenons d'emblée à en préciser le sens : la cinérgie, foyer de la dimension cinématographique, se loge entre mots, pierres et images, sujet, lieux et un avanttexte où les « films d'auteurs » occupent une place plus importante que les œuvres littéraires. La dimension cinématographique, et l'énergie qui en serait l'effet et la cause, sont ce qui se décrit, s'enregistre et se projette, le plus souvent simultanément, réversiblement, grâce à une cinécriture la 
plus à même d'écrire et de voir, de faire voir et de toucher la matière à la fois emmurée et mouvante, circonscrite et éclatée, d'une ville comme Fès. La cinécriture est en lien avec la notion de « caméra-stylo ${ }^{1}$ » dont la filiation théorique est revendiquée par les Cahiers du cinéma ${ }^{2}$. La «camérastylo » tend concrètement vers ce qui lève le poids excessif du discours verbal sur l'image et sa plasticité intrinsèque, autonome, et ce, aussi bien au cinéma qu'en littérature. Le recueil Aya dans les villes atteste qu'une « bifurcation » (celle du septième art) a bien failli avoir lieu dans le cadre des « cultures d'islam », notamment dans la culture mystique. Des gestes, comme ceux des calligraphes, constituent l'énergie émettrice et réceptrice d'une cinécriture, voire d'une pictoécriture et d'une photoécriture ${ }^{3}$; même principe, mais cette fois-ci respectivement en lien avec la peinture et la photographie.

Une fois ces précisions énoncées, posons-nous cette question : quelle est, au juste, la matière d'un bâti ? Peut-on délimiter en termes catégoriques les contours d'un espace urbain? En cultures d'islam, le syntagme madinâ (« ville »), met en avant la notion d'endettement : madîn(a) qui signifie littéralement « endetté(e) », dérivant de dâyn, « dette ». Dans le dictionnaire Lissân Al Arab, un énoncé tel que ibnûamatîn («fils d'une esclave ») est synonyme d'ibnumadinâ (fils de madinâ). Le syntagme bâb/abwâb («porte(s)»), utilisé au sens de « chapitre », indique également logos et topos qui, renvoyant peu ou prou à la notion de syntaxe, annâhwh, laquelle présuppose un processus d'orientation, wijhâ, qui dérive, entre autres, de wajh et wijhât nadâr (« visage » et « point de vue »). Sans doute, ces précisions lexicales sont binaires et souvent exclusives. Cela contraint corpus et corps, langue et œil ou encore ville, et implique un balisage sans faute ni erreur. Quand bien même contraint, ledit sujet, enfant/fatâ, s'empêche-t-il pour autant de sauter par-dessus murs et « remparts ${ }^{4} »$ ? Confinée à un statut

1. Alexandre Astuc, "Naissance d'une nouvelle avant-garde, la caméra-stylo » [Écran français, $\left.\mathrm{n}^{\circ} 144,1948\right]$, Trafic, $\mathrm{n}^{\circ} 3$, 1992, p. 147-151.

2. Abdelwahab Meddeb y a publié bon nombre d'articles et de comptes rendus.

3. Cf. notre article « Atelier de mémoire postcoloniale : le photographique », dans K. Zekri et A. Ouyachchi, Études postcoloniales : théorie, littérature, art, Meknès, Publications de la Faculté des Lettres de Meknès, 2011, p. 157-175.

4. Écrivain bilingue, Abdelfattah Kilito, est attentif à ce qui sauve du binarisme (intramuros vs extramuros). Il écrit dans un fragment d'autoportrait intitulé «Le rempart»: «Le voyage devint quotidien, de la médina vers l'au-delà du rempart, de l'espace familier à l'espace étranger. Voyage, aussi, du registre de l'oral à celui de l'écrit [...]. En dehors de l'institution scolaire, le français n'avait pas cours. Entre eux, les élèves ne le parlaient pas, et à la maison, il était proscrit. C'était la langue de la séparation : pour la première fois peut-être dans l'histoire du Maroc, les enfants avaient reçu une langue inconnue de leurs parents. Il fallait sortir de la maison, quitter la médina, aller au-delà des remparts et fouler une terre où je ne m'étais jamais aventuré auparavant, celle de la ville nouvelle. » (Adelfattah Kilito, «Le rempart », dans A. Belhabib Tlemcani (dir.), Littérature et altérité, Rabat, OKAD, 2009, p. 18.) 
strictement utilitaire et fonctionnel, l'urbanité n'a-t-elle pas partie liée à une matière faite de pierres, de lettres mais aussi d'images fixes et mobiles qui se déploient en un ensemble de figures prises dans un espace mouvant, comme une architec(x)ture en devenir ${ }^{5}$ ?

Au sujet d'une urbanité comme celle de la médina, il y a lieu de relever qu'une acception occidentalo-centriste la réduit à un état de désordre irrécupérable, géométrie déréglée, irrationnelle, privée de toute perspective. Voici ce qu'écrit à ce propos Abdelwahab Meddeb, auteur du recueil de récits intitulé Aya dans les villes ${ }^{6}$ sur lequel nous nous pencherons ici :

Quoi de plus antithétique en effet que l'urbanisme romain et islamique? L'un applique selon un souci maniaque l'idéalité orthogonale; l'autre propose un écheveau qui ferait perdre le sens à l'avisée Ariane. À la rigueur des rues qui, en toute symétrie, ne se croisent qu'en angles droits, s'oppose le plus touffu des labyrinthes. Je vous épargnerai les conclusions ineptes qui ont été tirées de ce constat, attribuant la raison géométrique aux maitres du damier et le désordre de l'improvisation à ceux qui laissent croître le dédale comme destiné à dérouter l'étranger incapable de discerner entre les rues ouvertes et les impasses. S'y ajoute le sentiment d'angoisse qu'instaure la feinte qui interrompt l'élan de la marche lorsque, dans une voie apparemment passante, vous êtes obligé de revenir sur vos pas après vous être confronté au cul-de-sac. Mais la vérité qui structure la ville islamique est plus complexe. Les principes fonctionnels de l'urbanisme romain sont tout à fait présents. Seulement le maître d'œuvre en islam refuse de les systématiser. Chaque cité est fondée sur la croisée du cardo et du decumanus; ceux-ci sont souvent réalisés à travers deux parallèles qui serpentent et qui conduisent à des portes s'accordant avec les points cardinaux. Et dans la zone centrale de leur rencontre se déploient les fonctions qu'accueille le forum : le temple et le marché (où le négociant côtoie l'artisan), parfois doublés du palais ou de la citadelle ${ }^{7}$.

Parallèlement aux partis pris réflexifs exprimés ci-dessus, ce sont notamment les récits qui nous semblent répondre le mieux aux discours exogènes autant qu'endogènes réduisant l'urbanité de la médina à une entité tantôt chaotique tantôt emmurée. Les récits de voyage de celui que

5. En intitulant un de ses recueils poétiques Matière des oiseaux (Saint-Clément-deRivière, Fata Morgana, 2001), Abdelwahab Meddeb rend un hommage à peine voilé au titre d'un essai de Gérard Genette sur Proust intitulé « Matière de Venise » (Figures IV, Paris, Seuil, p. 256) qui, dans une version posthume de La Prisonnière, réécrit de façon identique et différente les séquences relatives à cette ville. Ce retour de l'œuvre sur une même « adresse » et sur elle-même rend possible un jeu de rapprochements et de substitutions entre notamment ville « en dérive », enfance, Mille et une nuits, et tapis volant. Nous verrons, qu'Abdelwahab Meddeb, procédant à coups d'impli-citations et de réminiscences, con-fond, lui, mémoires, villes, livres et films.

6. Abdelwahab Meddeb, Aya dans les villes, Saint-Clément-de-Rivière, Fata Morgana, 1999.

7. Voir son essai intitulé Islam, la part de l'universel, Paris, Adpf, 2003, p. 13-14. 
nous nommerons « héros écrivain ${ }^{8}$ », se qualifiant, ici et là, de « nouveau pèlerin », refusant de visiter les lieux bâtis, ceux dont il est le « passager » autrement qu'à travers leur permanente capacité à « l'émouvoir » et, surtout, à « démentir » le « dogmatisme le plus vif [qui] efface les durées ${ }^{9}$ ». Contrairement à ce qu'on pourrait penser, le « nouveau pèlerin » est dans le « non agir » où il « jubile », lit-on dans le premier récit intitulé « La tache blanche » car « [il se] sent en mille lieux ensemble, et telle simultanéité [le] divise sans [1'] exiler du présent ${ }^{10} »$. Ruinant tout binarisme, à quelque niveau que ce soit, le « héros écrivain » se meut, se déplace et s'égare en étant constamment lié et séparé par l'expérience de l'entre-deux. Ce type d'interzone est ce qui nous semble correspondre à la logique poétique des lieux, mi-lieux, qui, agis et agissants, forment la communauté d'adresses fixes et mobiles, matérielles et immatérielles, factuelles, verbales et iconiques et, qui plus est, s'avèrent capables d' " ouvrir des questions ${ }^{11}$ » en territoires imprégnés de ce que Meddeb lui-même appelle « cultures d'islam ».

Nous ferons nôtre l'hypothèse selon laquelle une poétique comme celle qui structure des écrits hybrides et fragmentaires d'Abdelwahab Meddeb relève moins de l'architecture et de la peinture que de l'art de l'organisation du temps dans l'espace : la cinématographie. Dans un passage de Talismano, son premier roman, un glissement s'opère vers ce que nous nommerons une cinérgie. Il s'agit du « roman » d'une déambulation dans les villes du Maghreb, laquelle ne cesse de recommencer, différemment, dans les fragments de ce qui a trait au même livre, à la même architecture (ville) autant qu'à la même cinématographie (film) inachevée :

Telle laideur envahit ces villes alors que jadis les exigeants ne dédiaient par trait d'union que des maintiens désarticulant peut-être mais courtisant sans cesse la beauté qui s'infiltrait à l'aube avec la lumière qui savait jouer comme elle voulait infinie variété à colorer insensiblement les corps de jour. Tandis que maintenant telles villes ignorent l'aurore : elles collaborent à la nouveauté universelle corrompue par perversion de néophyte. Et l'enfant que je fus était témoin de l'aurore nuptiale qui luisait sur sa ville et qui participait à l'éveil des sens, à l'emprise de l'univers, à la tentation de l'impossible, très tôt la musique s'approchait de nous pour nous ravir des craintes et des pleurs; à être fascinés autant qu'effrayés par les Bûsa'dia qui se promenaient bavants, écumants, noirs, vénérables vieillards portant des chapeaux décorés de plumes de corbeaux, chemise rouge, crotales, incendiaient l'oreille de mes dix ans ${ }^{12}$.

8. Auto-qualification formulée par un «allié substantiel », Claude Ollier, dans son livre d'entretiens Les Liens d'espace (1970-1980), Paris, Flammarion, 1992.

9. Ibid.

10. Abdelwahab Meddeb, « La tache blanche », Aya dans les villes, op. cit., p. 30.

11. Abdelwahab Meddeb, «L'Islam de fonds », entretien avec Aurèle Hadj-Lararo, Les Inrockuptibles, $\mathrm{n}^{\circ} 210,1999$.

12. Abdelwahab Meddebb, Talismano, Paris, Christian Bourgois, 1987, p. 69. 
Bien que menacée de disparition, captée et projetée à l'air libre, une lumière « aurorale » réussit ainsi, via des « villes » à l'abri de la « nouveauté universelle ", à être une " infinie variété à colorer insensiblement les corps »; se révélant dans le studio au même titre que dans la chambre noire (claire) que sont les rues, une suite d'images « carnavalesques » qui, tout en « éveillant les sens », ne cessent d'y faire «trait d'union » entre fixité et mobilité, loin de la moindre distinction entre souvenir, geste, voix, son, musique, ciel et mur. Dès le titre du recueil Aya dans les villes, dont trois des onze récits sont consacrés à la médina de Fès (« Retour à Fès », «L'amour, l'après-midi » et « Hammam fassi $\left.{ }^{13} »\right)$, les intitulés pointent les contours d'une réelle hybridité. Celle-ci s'apparente à une matière conjuguant, outre l'allusion au Coran et au célèbre roman de Lewis Caroll Alice aux pays des merveilles, un clin d'œil au film de Wim Wenders intitulé Alice dans les villes $(1973)^{14}$. Dans « Retour à Fès », le syntagme initial nous dit le retour au « lieu » de réécriture qu'est Fès dans Talismano. Il s'agit du travail que mène l'œuvre sur elle-même : l'auto-réécriture des « fragments » relatifs à la même ville dans Talismano et après cette œuvre. Dans le deuxième titre du triptyque, le substantif « amour » dit de son côté l'état de l'éternel recommencement inhérent au désir du sujet agi autant qu'agissant dans l'espace et les instantanés d'une « après-midi » du mois «d'avril » ${ }^{15}$. Aya, en tant que prénom de personnage, désigne également le(s) fragment(s) contenu(s) dans chaque verset coranique, sûra, dont les liens étymologiques avec le verbe sawwâra (" emmurer »), disent la sacralité des " Murs du Livre », abri et foyer par excellence des versets $(A y \hat{a} t)$. À la fois réécriture et autoréécriture, le recueil Aya dans les villes ainsi que l'ensemble de l'œuvre de Meddeb, sont liés moins à un avant-texte immédiatement identifiable, qu'à un aller-retour entre un avant-texte et un après-texte poreux, ouvert et mobile où, parallèlement aux dimensions coranique et littéraire, il y a la part du « cinématographique ». Le langage et le métalangage historicisés,

13. «Hammam fassi » fut publié au départ dans le numéro spécial de la revue Europe (no 702, 1987, p. 141-143) consacré à «La littérature tunisienne ». Ce lieu, le hammam, à mesure qu'il est visité, revisité, donne et reçoit une épaisseur originelle; il est, à chaque fois, « fragment » d'une œuvre dont l'unité est ouverte, inachevée.

14. Ce long métrage est le « récit de voyage » d'une fille, Alice, accompagnant son père adoptif en Europe et aux États-Unis. En prenant des photos le long de leur périple, le film nous fait voir à quel point aucun des « instantanés » ne ressemble à l'évidence vécue, les merveilles qu'est l'hyperréalité traversée. Arriver à identifier l'actrice à même d'incarner le rôle écrit, scénarisé, tel est le pari, toujours entravé, différé, du protagoniste, «héros cinéaste » Niccolo, alter ego du scénariste et metteur en scène du long métrage intitulé Identification d'une femme, Michelangelo Antonioni (1981). Titre, qui est, notons-le, implicité dans l'un des onze récits du recueil en question ici : "L'identification d'une maison » (p. 123-131) où il s'agit d'une enquête menée par le héros écrivain à partir des ruines romaines, non loin de Tunis, sa ville natale, là où il mène une tentative d'identification d'un temps dont les « ruines » sont à portée de main et lointaines en même temps.

15. Abdelwahab Meddebb, «L'amour, l'après-midi », Aya dans les villes, op. cit., p. 55. 
codifiés et, bien souvent, surcodés, déterminent la cinérgie d'un «monde [qui] résiste à se laisser mettre en boîte par la reproduction technique ${ }^{16} »$ de quelque nature que ce soit, « machine à écrire » ou « caméra ».

La minceur diégétique du triptyque - arrivée par train à Fès, séance au hammam et scène d'amour dans une chambre d'hôtel - se verra compensée, de bout en bout, par une explosivité plastique, une " expérience des limites ${ }^{17} \gg$ où un ensemble de micro-lieux sont fragments de temps multiples et simultanés. À travers la texture, la pellicule et le studio à l'air libre qu'est la médina, le héros écrivain et cinéphile n'aura de cesse de se frotter à ce qui ne cesse d' " éclater », d' " exploser » à travers des images et des sons composant des arborescences en mouvement et, court-circuitant la moindre séparation entre dehors et dedans, sujet et objet, fatâ et madîna. Quand bien même abandonnée, ou emmurée, cette cinérgie, matière-prisedans-le-temps, participe dans les trois récits et dans l'ensemble du recueil d'un processus qui « désoriente les sens ${ }^{18}$ » en « restant, résistant ${ }^{19}$ » à toute volonté de reproduction à l'identique. Il s'agit d'un état de débordement qui finit, à l'occasion de chaque visite, par ouvrir la voie à ce qui permet d' « autonomiser la musicalité et la plasticité de l'enchaînement des images au bénéfice d'une acception plus physique et plus sensorielle de l'effet cinéma $^{20} \gg$ et/ou de l'effet-littérature de la ville.

16. Youssef Ishagpour, D'une image à l'autre : la représentation dans le cinéma d'aujourd'hui, Paris, Denoël, Gonthier, 1982, p. 183-184.

17. Aux débuts des années 1980, à l'issue d'un premier voyage au Maroc, Abdelwahab Meddeb, écrivain cinéphile, passionné d'architecture, se disant encore "néophyte en modernité », avance dans un entretien intitulé « Dons et excès », publié dans la revue Europe, citée ci-dessus : « J'étais tout entier à mon apprentissage européen qui engageait le corps et l'esprit en des aires régies par des mécanismes éloignés de l'instinct rivé au sang et à la sueur. J'étais néophyte en modernité, incapable d'apprécier les vertus de "l'archaïsme". J'étais dans l'approche d'une plastique plus orientée vers la mesure» (p. 18-21).

18. Ibid., p. 51.

19. Un tel état est effet et cause en même temps d'une relation de tension triangulaire qu'arpentent deux cinéastes, Jean-Marie Straub et Danielle Huillet pour qui le «plan, atome de base de leur cinéma, est produit au sens de restance d'une triple résistance: des textes aux corps, des lieux aux textes et des corps aux lieux. Et vice versa » (Serge Daney, «Le plan straubien », Les Cahiers du cinéma, n 305, 1979, p. 5). Meddeb publie un article relatif à un de leurs films, Chiens du Sinaï (1976). Voir Abdelwahab Meddeb, «La guerre des six jours et le délire occidental », Libération, 17 mai 1979.

20. Dominique Païni, «Faire violence : à propos du trafic des cassettes vidéo », Cahiers $d u$ Cinéma, ${ }^{\circ}$ 524, Paris, 1998, p. 92-97. L'auteur avance ici dans le sillage de Roland Barthes qui dit s'ennuyer face au « défilement métonymique » de la projection cinématographique empêchant la relation de « pensivité » que permet le « photogramme ». Lire son essai « Le troisième sens », dans L'Obvie et l'Obtus, Paris, Seuil, 1981. À l'ère du magnétoscope, les écrivains cinéphiles vont opérer des retours aux films et à leurs photogrammes en tant que foyers de détails non vus ou mal vus par le défilement standard (24 images par seconde). La réécriture-redéfilement chez Meddeb est en même temps prélèvement et agrandissement, ralentissement et accélération du déjà bâti, écrit et filmé. Point de séparation entre pierres, mots et images. 
Dans Fès ou les bourgeois de l'islam, relatant dès l'incipit leur arrivée en 1924 devant la médina en compagnie du général Lyautey, les frères Tharaud n'y ont vu, d'après leurs propres termes, que "poussière ", «bêtes » et « humains affolés » par l'avancée de leur " voiture klaxonnant $^{21} »$. Leur vue, évasive et superficielle, des paysages et des visages, accompagnée par un dispositif technique tel que la voiture ou le char, le canon, l'horloge, l'avion, la machine à écrire, l'imprimerie et la caméra, participent d'une logique et d'un pouvoir de « franchissement des frontières en temps et espaces réels, véhicules de l'urbanité en tous lieux homogénéisant des territoires ${ }^{22} »$. Les frères Tharaud, appréhendant la médina comme signe d'un « Orient en ruines », la réduisent au décor d'un spectacle anecdotique ou exotique. La cinécriture à l'œuvre dans le triptyque agit en tant que réécriture et/ou redéfilement d'un déjà bâti-écrit-filmé. Les "indigènes », longtemps acculés à poser en tant que silhouettes d'ombre, couchées ou affolées, resurgissent dans Aya dans les villes comme des visages et des paysages à la fois cadrés et incadrables, " griffant » le « scénario » préétabli de sorte qu'un écart, un " abîme " ne manque de s'installer entre la « représentation» et le « réel», image d'un entre-bords ou entre-deux, foyer d'un « troisième œil », d'une « voyance » et des « visions ${ }^{23}$ » attestant la « restance/résistance » à toute identification immédiate et à toute reproduction définitive ${ }^{24}$. Nous assistons au retour du/au «temps perdu » par le regard distant, lointain et panoramique des écrivains et des filmeurs voyageurs empressés, expéditifs. Les intérieurs que sont le compartiment, le hammam ou la chambre d'hôtel, dans lesquels sont successivement « localisés » les trois récits, ne cessent d'être excédés, débordés par ce qui grouille, « explose », en eux, hors d'eux. Et la cinécriture est cela

21. Jérôme et Jean Tharaud, Fès ou les bourgeois de l'islam, Paris, Plon, 1930, p. 11-16.

22. Odilon Cabat, "L'invasion du digital», Architecture aujourd'hui, n 374, 2009, p. 39-52.

23. Abdelwahab Meddeb, Aya dans les villes, op. cit., respectivement p. 94, p. 45 et p. 51. 24. Dans un film des frères Lumière intitulé Coolies à Saïgon, le filmé ne concorde pas totalement avec l'intention du filmeur: " une vue coloniale type, vantant les bienfaits de la civilisation occidentale et son acceptation par les indigènes. Un défilé de Vietnamiens, toujours en diagonale dans le plan, passe en citant un lourd rouleau qui aplanit une route, la route du progrès. Mais le plan dure, dure encore, et derrière le rouleau, on voit des petits garçons qui jouent, un enfant qui porte de l'eau et vient vers la caméra, qui la regarde, s'en va, puis une brouette qui passe, poussée par un adulte. Là, dans cette série d'accidents, l'image raconte une autre histoire de l'épopée coloniale : c'est la vie des gens qui vivent tout à fait indifférents aux prétendus progrès qu'apporte la civilisation française, qui ont leur vie autonome, une force d'inertie formidable par rapport au discours officiel, une force qui passe par l'accident de l'image. Tout simplement parce que la caméra est posée et qu'elle enregistre » (André S. Labarthe, «L'espace, le temps, le hasard, le noir, la neige », Cahiers du cinéma, $\mathrm{n}^{\circ} 489,1995$, p. 51). Cette part d'accident et de hasard est ce qui rend le film ouvert sur l'opération de redéfilement, la réécriture et, par là même, le processus d'interprétation et de réinterprétation sans fin. 
même qui, continuellement, en donne et en reçoit les éclats et les éclosions printaniers; déploiement d'un "regard fixe et mouvant», de «visions qui s'accélèrent ${ }^{25} »$, elle est à l'image d'un continuel effort d'adaptation, de mise au point. Stylo-caméra à l'épaule et stylo magnétoscope en tête, le héros écrivain cinéphile entreprend la mise en forme de son livre-film « sur la route » comme narrativité continuellement « retardée », fragmentée, éclatée :

Comme chaque fois, ton regard se décompose, tu apprivoises le vertige devant ces réseaux d'arabesques dont les creux sont coloriés par des teintes pastel bleues, jaunes, vertes, sous la bénédiction des carrés calligraphiques qui affinent les lettres et les orientent vers une déroute où la flore se dissout et se reconstitue dans une géométrie en mutation, emportée en sa fixité même par une vocation cinétique ${ }^{26}$.

Il s'agit de trois récits poétiques où une logique de «correspondances », de « répétitions et de variations ${ }^{27}{ }^{7}$ s'enregistre et se projette en tant que force centripète et centrifuge, contenue et « explosive». Chacun des trois récits constitue une illustration éloquente de cet état interstitiel, « auroral », évoqué auparavant à partir de Talismano, qui recommence ici en passant outre la moindre limite, la moindre clôture tant en amont (incipit) qu'en aval (clausule) : Dans « Retour à Fès », l'incipit est : " Léger comme un drap qui claque au vent, je suis porté par la vitesse »; et la clausule donne : « je rêve que je marche dans les premières lueurs de l'aube »; Dans « Hammam fassi », l'incipit est : « D'entrée le patron vous offre un sebsi, la pipe circule parmi les hommes, l'odeur du kif pénètre mes narines, l'herbe sèche se consume »; la clausule en revanche est rédigée ainsi :

Je porte en moi le rayon éclatant et l'écho qui résonne, le sexe en éveil à la rencontre d'Aya, dans la perspective des touches d'amour, voyage céleste dans la pleine lumière et la musique des sphères.

Dans « L'amour, l'après-midi », l'incipit donne : « Comme quand on vient de faire l'amour, l'après-midi, en avril, c'est déjà la canicule, du balcon, à flanc de colline, intramuros, le regard est attiré par le vide, les pensées flottent sur les toits »; la clausule est rédigée ainsi :

La dame vous presse, vous caresse, elle vous convie à vous reposer dans la géométrie de l'œil et de la lèvre, fiole contre fiole, poudre noire, poudre rouge, antimoine et coquelicot, cierges géants allumés sur la tombe du saint, la ville explose, c'est le crépuscule, les appels crépitent.

Chaque ouverture ou fermeture est ainsi trace de ce qui ne cesse d'être « aube » ou « crépuscule ». C'est l'état d'une ville où l'énergie intérieure

25. Abdelwahab Meddeb, Aya dans les villes, op. cit., respectivement p. 47 et p. 51.

26. Ibid., p. 68.

27. Ibid., respectivement p. 48 et p. 19. 
(compartiment, hammam et chambre) et extérieure (ciel, cosmos) opèrent selon une logique d' « accouplement», de réversibilité et d'alternance. C'est le cas d'un train qui permet à une « superbe campagne d'entrer dans le bourg 》 et de surgir en « circul[ant]» dans et hors le compartiment et sa fenêtre, devenus « chambre » d'enregistrement, de projection et d'arrêts sur image allant d'un « arbre » aux « veines d'un vieillard hiératique ». Il s'agit d'une sortie et/ou d'une « entrée en matière » augurant de ce qui ne cesse de traverser et déborder phrases, champs, cadres ou écrans :

Mon regard s'immobilise devant un arbre qui bouge à peine, en face de moi, un vieillard hiératique, aussi impassible que l'arbre que je viens de croiser, de ses voyantes veines monte la sève du sentiment, il s'en dégage une rectitude qui abolit les obstacles entravant la circulation dans le monde [...], la couronne qui ceint les ombres des arbres les arrache du sol et les élève dans une lévitation accentuée par la vitesse, chaque arbre ainsi défini par son ombre, s'incarcère dans un contour qui l'isole, son individualité s'affirme avec violence qui le coupe du monde, chaque arbre est un, il est en soi, il ne se confond pas avec la masse d'enclos, d'un bosquet, d'un bois, l'effet de la distance est suspendu par l'éveil de la conscience, l'arbre est unique, inconfondable, il participe au rythme qu'engendre le nombre ${ }^{28}$.

À la fois détail et totalité, unicité et multiplicité, «l'arbre » permet de voir à quel point le regard est constamment pris entre ce qui «incarcère » et ce qui fait « circuler», ce qui se " disperse » et ce qui se « concentre» selon la logique de l'a-grammaire, la seule à même de "peser » le nombre incalculable de « détails en émeute ${ }^{29} »$ excédant sans merci le moindre « contour qui isole», le transformant en « lambeaux d'un aloès déchiré par les mains acérées du vent ${ }^{30}$ » qui « circule dans toutes les directions », pareil à la lumière qui « inonde ${ }^{31} »$ :

Comme quand on vient de faire l'amour, l'après-midi, en avril, c'est déjà la canicule, intramuros, le regard est attiré par le vide, les pensées flottent sur les toits, naviguant de contrée en contrée, dans le pays de la sueur, ton corps est collé à un drap de poussière, sous un ciel brumeux, qui ouvre la ville à son $\operatorname{ardeur}^{32}$.

Entremêlement du dehors et du dedans, des vues aériennes et des contreplongées, de/à l'intérieur, la tâche du héros écrivain cinéphile révèle

28. Abdelwahab Meddeb, Aya dans les villes, op. cit., p. 41.

29. Les «détails font émeute»: formule de Baudelaire reprise par Daniel Arasse dans Le Détail : pour une histoire rapprochée de la peinture (Paris, Flammarion, 1998) où l'auteur relève et agrandit ce qui fait «tache blanche» dans les toiles : écart, hasard ou oubli, en dépit des choix dits conscients, centripètes, du Peintre Auteur. Force centrifuge, certains détails sont désir ou poussée à la fois cadrable et in-cadrable.

30. Abdelwahab Meddeb, Aya dans les villes, op. cit., p. 42.

31. Ibid., p. 57.

32. Ibid., p. 55. 
l'urbanité en tant qu' « ardeur », plénitude d'un «vide» du visage d'un jeune qui, quand bien même cadré de près, reste " proposition » lisible et illisible, dicible et indicible, "enfilade » d'images transparentes et opaques. Une telle ambivalence ne manque pas de faire écho au récit intitulé « Chouette et djinns » où, lors d'un moussem, pèlerinage, la foule dévisage le « héros écrivain » à l'image d'un étrange étranger :

À ton entrée, les interrogations s'aiguisent, qui est-il? D'où vient-il? Il parle arabe, il a un accent étranger, serait-ce un espion? ou un chercheur? laissez-le, non, arrêtez-le, ta main relève ta chevelure, apparaissent tes yeux clairs, changeants, intenses, marqués par une peur essentielle qui n'est pas réactivée par la conjoncture, la mèche obscurcit ton visage et rend ton regard ancien, ta chemise ample à l'indienne ajoute à ton étrangeté, $[\ldots]$, ta présence est une intrusion qui perturbe la séance, la foule est stupéfaite, elle ne veut pas être troublée en sa célébration du saint qui apporta l'Islam à ses ancêtres, qui es-tu pour oser les provoquer en leur communion avec le commencement et l'origine? L'entrée est interdite aux mécréants, ta réponse coranique dans la lettre fuse : «Leur signe est dans leur visage ${ }^{33}$. "

Visage et face, n'y coïncidant pas, forment un texte, au final, indéchiffrable aux yeux d'une « foule stupéfaite » préférant voir-lire le réel en tant que catégorie aux contours circonscrits, évidents. Aux antipodes d'une acception figée, identitaire, la visagéité y est plis, strates divers et mouvants. Dans un récit déjà évoqué, "Tache blanche », sur le site de Pétra, en dépit d'une tranquillité convenue, impassible, le visage d'un chauffeur de taxi se révèle tout aussi secret voire « flamme insaisissable » :

Dans la représentation qu'il a de lui-même, il ajoute son nom à la longue liste des hommes rencontrés dans ce pays et prétendant avoir participé à la bataille de Karamé où les Palestiniens avaient résisté aux Israéliens. Il fut de nouveau mobilisé pendant la guerre du Golfe [...]. Il nous rappelle enfin l'allégeance de sa tribu au trône hachémite, parachevant le portrait de l'homme tranquille qu'il voudrait être; l'attirance pour la fantaisie et l'aventure ne le détourne pas des normes. Mais un doute inconscient trouble son regard et avive son visage d'une flamme insaisissable ${ }^{34}$.

Loin de tout partage, un état aussi cinétique déborde les contours du moindre matériau, qu'il se nomme visage, minaret, mausolée ou rosace, et dont la moindre saisie exhaustive se voit en réalité mise en échec :

Comme chaque fois, ton regard se décompose, tu apprivoises le vertige devant ces réseaux d'arabesques dont les creux sont coloriés par des teintes pastel bleues, jaunes, vertes, sous la bénédiction des carrés calligraphiques qui affinent les lettres et les orientent vers une déroute où la flore se dissout

33. Coran, 68-69.

34. Abdelwahab Meddeb, Aya dans les villes, op. cit., p. 24. 
et se reconstitue dans une géométrie en mutation, emportée en sa fixité même par une vocation cinétique ${ }^{35}$ ?

$\mathrm{Au}$ détour d'une venelle, le couple assiste, dans le dernier récit du triptyque, à une scène atypique versant dans la variance de la même logique de résistance. Il s'agit de la présence d'une «nubile » vouée au statut de « victime sacrificielle » qui révèle, par l'intermédiaire d'un léger travelling, suivi d'un gros plan, un être véritablement farouche, refusant avec force le mariage arrangé que sa mère négocie avec un « enhardi vieillard ». C'est le retour de la figure d'une intraitable « indigène » faisant face au tutorat, au « protectorat » :

Dans l'échoppe, je suppute que le vieillard et la vieille négocient les termes de l'échange pour une noce rassemblant l'enhardi vieillard propriétaire et la progéniture de la vielle démunie, nubile en léger retrait, gardant le silence, aiguisant une détermination qui ne signale pas l'abattement de la victime vouée à l'autel du sacrifice, mais l'assurance de la future jeune dame douairière ${ }^{36}$.

À l'issue d'une courte escapade, le couple rejoint sa chambre ému, dérouté, mais il ne tarde guère à se retrouver au cœur d'une heureuse et fertile con-fusion plastique obligeant au retrait de toute mollesse, de toute anesthésie sensitive. Il s'agit d'une densité où notamment l'aura d'un matériau tel que la voix se découvre littéralement détrônée :

Les voix des muezzins se chevauchent, se répondent, à une minute près, le chœur répète les mêmes formules se relayant en un décalage d'infime intervalle, qui apporte l'émoi en entretenant tel différé, que je déguste dans le labyrinthe de la volupté, sans cesse frôlée, toujours reculée, réparant le discord des énergies, jusqu'à éclater de concert et faire se tordre les corps crispés avant de se relâcher au comble des nerfs, spectateurs enflammés de leurs propres rôles mesurant leur violence, similaire à celle qu'entraîne l'éclosion des fleurs, dans la satisfaction qui rassérène avant d'ouvrir sur le vide, les membres gourds encore immergés dans le bain de la jouissance, je suis rattrapé par la prestation retardataire d'un vieux muezzin, ultime déclaration, lointaine, solitaire, aiguë, cassée par l'effort, d'une personne s'époumonant pour être à la hauteur de la récitation, sans se faire aider par la machine qui amplifie la voix, la suture et la brouille ${ }^{37}$.

La force consonante et dissonante de la voix dans « Retour à Fès » et «L'amour, l'après-midi » fait que le tout, se chevauchant, s'interpénétrant, réussit à « embrouiller » micro, haut-parleur et cassette. Grâce à une réelle " polyphonie ${ }^{38}$ », nous assistons moins au passage du muet au parlant qu'à la réhabilitation du son émis et reçu comme matériau de nature 
éminemment « physique ${ }^{39}$ ». La médina se révèle bande-son et bande-image unes et multiples :

[...] le chauffeur de bain se saisit du dernier rayon du jour, brusque et furtif, pour en conter le trajet à l'oreille de son four, cône de terre, retenant son babil pour ne rien perdre des chuchotements de son confident, brouillés par les grincements et les étincelles des rémouleurs, lames mouillées glissant sur les roues de pierres qui tournent vite, crissement aiguisant le frisson, que n'apprivoise pas le spectacle de l'artisan cerclant ses seaux de bois et peignant leurs cerceaux de fer avec le lourd goudron de thuya, odeur qui enivre, [...], la ville explose, c'est le crépuscule, les appels crépitent ${ }^{40}$.

Élargissant son espace par-delà un binarisme conventionnel opposant culturel à cultuel, la plasticité polychromique et polyphonique réussit, non sans heurts, à réconcilier lettres, voix, sons et bruits, d'un côté, raisons et résons, de l'autre :

[...] les muezzins mêlent la dissonance aux consonances, le grave relaie l'aigu, l'un commence où l'autre finit, les phrases se croisent, se chevauchent, les noms s'agitent, comme les dés dans le gobelet, les mots s'enchevêtrent et se démêlent, ils s'éteignent, se rallument, avant de se perdre dans la cadence qui rameute les anges, atterrissant sur les fils noués entre les minarets, plongeant dans les puits de lumière, pour surprendre les enfants qui jouent aux billes, et éblouir les femmes qui plient le linge, et ranimer les flots du torrent encombré par les ordures, et chasser les démons qui fouillent dans les immondices, et pleurer sur le sort des amantes changées en chattes dont le nez est dévoré par la lèpre $[\ldots]^{41}$.

Matériau dissonant et consonant, la ville se déploie en tant que trame inextricable : « fils nouant », raccordant « anges » et « djinns », hasard et nécessité. Elle (la ville) cesse donc de « concilier des antithèses ${ }^{42}$ » selon une formule de Marcel Duchamp à qui un hommage est rendu au passage à travers la figure des «machines célibataires ». C'est, au fond, l'éloge de ce qui continue de carburer aux combustions « d'avant le charbon », c'està-dire, à l'ardeur, à la sueur, à l'eau et au sang : « Devant l'animation que connaît l'heure vespérale, dans la bousculade, disponibles et à l'affût, les machines célibataires entrent en connivence ${ }^{43}$. »

39. « Nous sommes de plus en plus convaincus que le domaine du son déborde celui de la culture. » Voir Ahmed Essayad, « Pour une écoute musicienne du monde », Kalima, horssérie $\mathrm{n}^{\circ} 1$, « Regards sur la culture marocaine », 1988, p. 128.

40. Abdelwahab Meddeb, Aya dans les villes, op. cit., p. 57.

41. Ibid., p. 55-56.

42. Éliane Formentelli, «Cantique-Duchamp ou la théorie du moteur invisible», dans Duchamp : Tradition de la rupture ou rupture de la tradition?, Paris, Union générale d'éditions, 1979 , p. 263-286.

43. Abdelwahab Meddeb, Aya dans les villes, op. cit., p. 46. 
Ces figures en mouvement agissent dans un incessant jeu de conjonction et de disjonction, liant et séparant matériaux disparates, irréconciliables et, néanmoins, compatibles, donnant lieu, au final, à des mariages, à des merveilles imprévisibles :

[...] la canicule qui devance la saison m'offre la vision des lézards buvant dans les aisselles des femmes, par la fenêtre entr'ouverte, je devine plus que je ne distingue la distribution du jaune et du gris sur les parois de la ville, dans l'intimité d'Aya nue, à travers les étapes du plaisir, dans la concurrence de la rumeur émanant de la médina, les cris de jouissance concordent avec la polyphonie qu'instaurent les appels de la prière annonçant une heure médiane, légèrement plus près du couchant que de midi $[\ldots]^{44}$.

$\mathrm{Au}$ cœur de ce "familier étrange ${ }^{45}$ ", surréel, celui de la "vision des lézards buvant dans les aisselles des femmes », un support tel que le manuscrit, qui, par moment, peut se voir, entre les mains sensibles d'un copiste « décentré par tous ses bords graphiques ${ }^{46}$ », le « ciel » de Fès se meut en page, écran et mur tout aussi «troués » par les « appels» et la « calligraphie fugace des hirondelles ${ }^{47}$ ». « Hantée par l'image ${ }^{48}$ » la lettre et la voix transgressent de la sorte la division inhérente à «l'alphabet » grâce au jeu de « miroirs ${ }^{49}$ » d'une urbanité dégagée de tout état d'emmurement-cadrage immuable, de toute autochtonie ou sédentarité exclusive :

La hantise de l'autre lieu où que je sois, ce désir fou de l'ubiquité, qui ne te laisse pas jouir du lieu où tu es, pourquoi t'orientes-tu vers un désir de Grenade, tu sais que le djinn n'est pas disponible ${ }^{50}$ ?

Où qu'il soit, le nouveau pèlerin chemine, erre et s'égare en tant qu'exilé heureux, se cherchant continuellement entre intra-muros et extra-muros, Orient et Occident, ne se reconnaissant, au final, qu'en « quelque chose de plus grand que l'univers pour [lui] ouvrir la poitrine » :

Villes, mille villes dans la ville, je me perds dans les villes de la ville, je n'ai pas besoin de quitter ses murs pour voyager, ainsi dans les rues, je reconstitue le labyrinthe, je marche en lui, je tourne et je retourne sur mes pas, dans la course des noms et des lettres et des graphes, alphabets de

44. Ibid., p. 44-45.

45. Ibid., p. 48.

46. Abdelkébir Khatibi, La Calligraphie arabe ou la Célébration de l'invisible [1976], Paris, Éditions du Chêne, 1997, p. 150.

47. Abdelwahab Meddeb, Aya dans les villes, op. cit., respectivement p. 55 et p. 54 .

48. Abdelkébir Khatibi, op. cit.

49. Abdelwahab Meddeb est le scénariste d'un film intitulé Miroirs de Tunis (1995, 60 min) réalisé en collaboration avec le borgésien Raul Ruiz. Le film et/ou le cinéma à l'œuvre dans le triptyque d'Aya dans les villes n'accepterait-il pas volontiers un titre général comme Miroirs (Portes ou Fenêtres) de Fès?

50. Abdelwahab Meddeb, Aya dans les villes, op. cit., p. 87. 
toutes sortes courants sur les enseignes, je m'avance à travers la barrière de pierres, parfois je n'en peux plus du règne de la pierre, et je me réfugie parmi le peuple du Sud dans l'immense ville du Nord, ne me conviennent pas la peau brune, les épices, la convivialité d'Islam ou d'Asie ou d'Afrique, ça ne suffit pas pour m'épargner l'étouffement, il me faut quelque chose de plus grand que l'univers pour m'ouvrir la poitrine, non, le voyage sur place, à la rencontre des peuples et des langues n'enlève pas l'oppression qui me pèse, qui m'écrase le corps, qui m'amène à éprouver de la difficulté pour respirer, comment dépasser cet état, comment gagner l'expansion après la contraction $^{51}$.

Rien ni personne ne semble capable de " guérir » le " nouveau pèlerin » de tant d' " excès » et de " dons », hormis la sensation du cosmique qui le traverse en quittant le hammam, la « Machine » d' « avant le charbon ${ }^{52}$ » où le « Je » voit son unité défaite, glissant d'une sensation à l'autre. Le masseur est le seul à même de lui « redonner forme, avec le gant noir, souple et rêche, [le] reconstituer, colmater [ses] fragments épars, dépister le peu qui reste du dépôt corrompu, [et] le rincer à grande eau, de quoi changer $[\mathrm{sa}]$ peau $^{53} »$ :

Je me lève, en effet, j'ai un corps neuf, je m'initie lentement à le faire fonctionner, comme s'il était en rodage, la marche est lente, précautionneuse, minutieuse, je renais après être passé par la mort, je reviens de l'autre monde, je redécouvre la rue sombre et déserte la nuit, je me sens aussi fragile qu'un personnage en cristal, aussi lumineux, aussi sonore, je porte en moi le rayon éclatant et l'écho qui résonne, le sexe en éveil à la rencontre d'Aya, dans la perspective des touches d'amour, voyage céleste dans la pleine lumière et la musique des sphères ${ }^{54}$.

En se logeant, grâce au hammam, dans « la vieille mémoire de [ses] os » et la " mémoire involontaire de [ses] membres », le nouveau pèlerin est celui qui cherche à voir, toucher, revoir, retoucher, ce qui permet de « neutraliser le temps ${ }^{55}$ » en le multipliant, en le dés-originant. L'ancêtre des thermes antiques n'est-ce pas ce « vestige architectural témoign[ant] du remploi islamique d'une fonction romaine destinée à exalter la jouissance et à célébrer le corps, le signe de l'acclimatation du monothéiste à l'héritage païen adaptant l'absolu, l'infini, l'éternité de son message divin à la précarité du corps humain ${ }^{56} »$ ? Strates multiples, souvent refoulées, entremêlant corps et corpus, antiquité et contemporanéité, le « hammam fassi » est ainsi

51. Ibid., p. 89.

52. Ibid., p. 34.

53. Ibid., p. 51.

54. Ibid., p. 52.

55. Ibid., repectivement p. 53, p. 50 et p. 62.

56. Abdelwahab Meddeb, «Le jardin des délices », Le Courrier de l'Unesco, vol. 50, n 4, 1997, p. 32-34, p. 32. 
« lieu de mémoire » au sens d'une adresse hyperbolique, allégorique car « intimement nouée de vie et de mort, de temps et d'éternité ${ }^{57}$ ».

Résumons-nous : le passionné insatiable, exilé et désenchanté heureux?, muni de son stylo-caméra-magnétoscope, se retrouve à Fès comme dans d'autres villes de « cultures d'islam » attentif aux champs de possibles que constitue une architec(x)tualité restée longtemps confinée, contrainte à (se) voir et à (se) lire en tant qu'emmurée, endettée et intouchable. Entre figuration et abstraction, document et fiction, plan-séquence et montage, le héros/écrivain/cinéphile ne cesse d'être à l'affût du « texte » et/ou du « film » et/ou de la «ville» qu' « enclot le lieu ${ }^{58}$ » et vice versa. Le livre, la ville et le film, désormais inextricablement mêlés, finissent par libérer l'altérité, les « dons et les excès » qui sont contenus en chacun d'eux. Les « détails », restés longtemps abandonnés, amarrés à la volonté de l'auteur, de l'architecte et de l'opérateur qui s'emploient, au fond, à éviter de voir une telle altérité voler de ses propres ailes, sont, de bout en bout, sujets à des interprétations incessantes, renouvelées. En médina ne serionsnous pas donc « épinglés » à une page, une pellicule, un mur et un écran?

Le pari poétique des trois récits (on peut étendre ce pari à l'ensemble du recueil), a sans doute partie liée à une matière-forme-prise-dans-letemps; laquelle, en rupture totale avec l'essentialisme identitaire relatif à " l'être » de "l'indigène » et du « sujet/Fatâ arabo-musulman », soulève une question cruciale : en médina, où sommes-nous au juste? Dans la mesure où tout surgit, resurgit sous forme d'une suite d'éclats et d'éclosions, voire de (di)visions, l'identitaire devient désormais cinérgie mettant à mal aussi bien la littérature que le cinéma dits de représentation ${ }^{59}$. Sans doute le type de cinéma qui sous-tend le recueil en général et le triptyque en particulier est-il du genre qui investit, non sans tension, l'entre-deux, entrebords, " abîme » risqué mais hautement fertile, inépuisable, se situant entre visibilité et invisibilité, lisibilité et illisibilité. Ensemble d'états corporels singuliers et pluriels, plutôt que corps individualisés, typifiés et folklorisés, la médina, agie et agissante, est aux antipodes du modèle architectural précolonial autant que colonial. Ce modèle voit sa grammaire et ses binarismes - intra-muros vs extra-muros, centre vs périphérie, ordre vs

57. Pierre Nora, Lieux de mémoire, Paris, Gallimard, 1995, p. 35. Le mérite d'un minaret ou d'un hammam ne réside-t-il pas ici dans le désir d'élargir le mémoriel dit, écrit, peint et filmé en langue française par-delà la carte et le calendrier de l'État-nation, quelle qu'en soit «l'origine »?

58. Pour Claude Ollier, «allié substantiel» de Meddeb, lui-même «héros écrivain cinéphile », ce dont il s'agit est de « tenter d'élucider enfin l'idée de “texte en amon" (délivrer le texte qu'enclot le secret du lieu) ». Voir Claude Ollier, « Projet de lettre à Jean Ricardou », dans Souvenirs écran, Paris, Gallimard, coll. « Cahiers du Cinéma », 1981, p. 24.

59. Notons à ce propos l'allusion, d'un côté, à la disparition des «salles de cinéma » se muant en « dépôt », de l'autre, au décor en carton-pâte du « film américain » (Abdelwahab Meddeb, Aya dans les villes, op. cit., respectivement p. 47 et p. 26). 
chaos, original vs copie - littéralement démantelés, mis en pièces. La densité plastique de/à l'œuvre est, de bout en bout, intériorité et extériorité rendant plus que difficile la volonté d'orienta(lisa)tion normative, prescriptive, du corps et du corpus, de l'œil et de la main. Ni ordre ni désordre absolus, la médina dit l'existence d'une vision globale et partielle, chaotique et ordonnée, perspectiviste et dédaléenne. C'est l' « écheveau » du « temps passionné ${ }^{60}$ » se déployant dans et hors la linéarité chronologique. Nomade et sédentaire, le héros/écrivain/cinéphile se meut et s'émeut dans un entredeux, « entre-bords », d'une étrangeté familière cadrée et décadrée, finie et infinie. Dé-folklorisée, la médina est ce qui re-surgit dans le triptyque à l'image d'un volcan dont les éruptions sont nommées pierres, phrases, images-sons non définitivement mont(r)és ni totalement coordonnés ou subordonnés. Les fragments d'une rosace, d'un visage, d'une voix, d'un ciel ou d'une peau sont, de bout en bout, image-son d'une surface profonde, dédale où des durées imprévues, imprévisibles, sont laves débordant mur, mot, page, rempart, cadre et champ.

Face à un architecte de la Résidence générale comme Henri Prost, pour qui la médina de Rabat est si "confuse, inintelligible et incontrôlable » qu'elle se condamne d'elle-même à la " ségrégation ${ }^{61} »$, il y a sans doute les « grammes mouvants ${ }^{62}$ » du triptyque d'Aya dans les villes, qui attestent de la résistance qui nous semble rendre possible, viable, les éléments d'une « histoire rapprochée de l'art» des « villes de la Ville» et, sans doute, des livres du Livre et des films du Film. Des éléments, ou « détails en émeute », qui sont à revisiter, réécrire et redéfiler en tant que matière d'un permanent processus d'interprétation réussissant sinon à faire exploser du moins à ébrécher, lézarder, le moindre support, volume et substance s'érigeant en bâti définitivement clos.

\author{
Abdelkrim Chiguer \\ Université Moulay Ismaïl - Meknès \\ Équipe d'études culturelles et postcoloniales
}

60. Pascal Bonitzer, qui a longuement réfléchi sur la logique des univers vertigineux, « sentiers ne menant nulle part» d'un Borges et d'un Hitchcock, écrit : «C'est moins le labyrinthe qui engendre le suspense que le suspense qui engendre un labyrinthe. Lorsque la ligne droite de la durée pure et indifférente est soudain passionnée par un élément plus ou moins abstrait, il se produit une espèce de densification du temps et de hérissement de l'espace, qui se couvre d'obstacles et se charge de méandres. Le labyrinthe, en ce sens, est un effet de la hâte, c'est du temps passionné » (Pascal Bonitzer, «La vision partielle », Cahiers du cinéma, $\mathrm{n}^{\circ} 301$, juin 1979, p. 35-41).

61. Abdelkébir Khatibi, «Urbanisme, idéologie et ségrégation : exemple de Rabat », dans Chemins de traverse : essais de sociologie, Rabat, Publications de l'Institut universitaire de la recherche scientifique, 2002, p. 210.

62. Severo Sarduy, « Tanger », Tel Quel, $\mathrm{n}^{\circ} 47,1971$, p. 86-88. 